\title{
Automatic detection of Long Method and God Class code smells through neural source code embeddings
}

This paper was downloaded from TechRxiv (https://www.techrxiv.org).

\section{LICENSE}

CC BY 4.0

SUBMISSION DATE / POSTED DATE

$15-12-2021 / 22-12-2021$

\section{CITATION}

Kovačević, Aleksandar; Slivka, Jelena; Vidaković, Dragan; Grujić, Katarina-Glorija; Luburić, Nikola; Prokić, Simona; et al. (2021): Automatic detection of Long Method and God Class code smells through neural source code embeddings. TechRxiv. Preprint. https://doi.org/10.36227/techrxiv.17206010.v1

$\mathrm{DOI}$ 


\title{
Automatic detection of Long Method and God Class code smells through neural source code
}

\author{
embeddings \\ Aleksandar Kovačević, Jelena Slivka, Dragan Vidaković, Katarina-Glorija Grujić, Nikola Luburić, Simona \\ Prokić, and Goran Sladić \\ kocha78@uns.ac.rs, slivkaje@uns.ac.rs, vdragan@uns.ac.rs, katarina.glorija@uns.ac.rs, \\ nikola.luburic@uns.ac.rs,simona.prokic@uns.ac.rs, sladicg@uns.ac.rs \\ Faculty of Technical Sciences, University of Novi Sad, Serbia
}

This work has been submitted to the Expert Systems with Applications journal for possible publication

\begin{abstract}
Code smells are structures in code that often have a negative impact on its quality. Manually detecting code smells is challenging and researchers proposed many automatic code smell detectors. Most of the studies propose detectors based on code metrics and heuristics. However, these studies have several limitations, including evaluating the detectors using small-scale case studies and an inconsistent experimental setting. Furthermore, heuristic-based detectors suffer from limitations that hinder their adoption in practice. Thus, researchers have recently started experimenting with machine learning (ML) based code smell detection.

This paper compares the performance of multiple ML-based code smell detection models against multiple traditionally employed metric-based heuristics for detection of God Class and Long Method code smells. We evaluate the effectiveness of different source code representations for machine learning: traditionally used code metrics and code embeddings (code2vec, code2seq, and CuBERT).

We perform our experiments on the large-scale, manually labeled MLCQ dataset. We consider the binary classification problem - we classify the code samples as smelly or non-smelly and use the F1measure of the minority (smell) class as a measure of performance. In our experiments, the ML classifier trained using CUBERT source code embeddings achieved the best performance for both God Class (Fmeasure of 0.53) and Long Method detection (F-measure of 0.75). With the help of a domain expert, we perform the error analysis to discuss the advantages of the CUBERT approach.

This study is the first to evaluate the effectiveness of pre-trained neural source code embeddings for code smell detection to the best of our knowledge. A secondary contribution of our study is the systematic evaluation of the effectiveness of multiple heuristic-based approaches on the same largescale, manually labeled MLCQ dataset.
\end{abstract}

Keywords: code smell detection, neural source code embeddings, code metrics, machine learning, software engineering 


\section{Introduction}

Code smells are structures in code that signal the need for refactoring to avoid degrading the software's quality attributes (Fowler, 2018). These code structures negatively impact the software products' maintainability, evolvability, and testability (Sharma \& Spinellis, 2018). Classes affected by code smells are change- and fault-prone (Khomh et al., 2012). Code smells negatively affect source code comprehension, decreasing developers' productivity (Sharma \& Spinellis, 2018). Thus, code smells increase the overall cost of software development (Sharma \& Spinellis, 2018), and software engineering experts agree that their removal leads to sustainable software development (Fowler, 2018; Sharma \& Spinellis, 2018; Martin, 2009).

Unfortunately, manually detecting code smells in practice is not easy. Many code smell definitions are vague, and refactoring decisions rely heavily on human intuition and experience (Fowler, 2018). Developers frequently need to detect smells in unfamiliar code, which is challenging (Hozano et al., 2018). A solution to this problem is developing the tools to automatically detect code smells in source code, and many such tools have been proposed (Azeem et al., 2019).

Most code smell detection tools use metric-based heuristics defined by the domain experts to determine whether a code snippet suffers from a particular smell (Azeem et al., 2019). Metric-based heuristic approaches calculate a set of source code metrics for a code snippet and compare the obtained metric values to predefined thresholds. However, as Azeem et al. (2019) pointed out, these approaches suffer from several limitations that prevent their adoption in practice. The most critical issues are the low agreement between the existing detectors and strong dependence on user-defined parameters, for which no principled way of defining has been devised. Machine Learning (ML) learn-by-example approach promises to solve these issues (Azeem et al., 2019).

A principal challenge in applying $M L$ algorithms to detecting source code issues is creating a vector representation of the analyzed source code snippet suitable for applying ML algorithms.

We recently witnessed a breakthrough in the Natural Language Processing (NLP) field with the introduction of Word Embeddings as a viable representation of linguistic items (Mikolov et al., 2013; Devlin et al., 2018). These vector representations of text capture both statistical and semantic information and are effective in various NLP tasks (Bakarov, 2018). As suggested by the "naturalness hypothesis" (Allamanis et al., 2018), source code has similar statistical properties as natural language. Substantial empirical proof for this hypothesis exists, as models initially developed for natural language proved highly effective in many source code analysis applications (Allamanis et al., 2018). Recently, inspired by the success of pre-trained contextual embeddings for natural languages, Kanade et al. proposed CUBERT (Kanade et al., 2020), a code understanding model inspired by the BERT model (Devlin et al., 2018) that holds state-of-the-art status in many natural-language understanding benchmarks. In their paper, Kanade et al. show that CUBERT displays state-of-the-art performance on multiple codeunderstanding tasks while requiring shorter training and fewer labeled examples than its alternatives. This paper will investigate the effectiveness of CUBERT on the problem of automatic code smell detection.

On the other hand, there are significant differences between the source code and natural language. In contrast to the natural language, source code is written as the formal language that presents minimal ambiguity, extensive re-use of identical "sentences," reduced robustness to minor changes, and contains 
long semantic units (Lozoya et al., 2021). For these reasons, representations that exploit code's structural nature have been proposed, such as code2vec (Alon et al., 2019) and code2Seq (Alon et al., 2018). These embeddings leverage the syntactic structure of programming languages by representing the code snippet as a set of compositional paths over its abstract syntax tree (AST). Code2vec and code2seq are regarded as the representative state-of-the-art among the contextual embeddings for source code (Rabin et al., 2021; Kang et al., 2019).

As Rabin et al. (2020) observed, few manually engineered features can perform very close to the higher dimensional code2vec embeddings. Thus, it is necessary to include handcrafted features as baselines. As source code metrics are frequently used handcrafted features for code smell detection (Azeem et al., 2019), we regard ML models trained using these features as baselines.

Our study aims to evaluate the effectiveness of pre-trained neural source code embeddings on the task of code smell detection. To this aim, experiment on the MLCQ dataset (Madeyski \& Lewowski, 2020), in which each code sample was manually examined for the presence and severity of code smells. In our experiments, we compare the performance of $\mathrm{ML}$ classifiers trained using the following source code vector representations:

- three neural source code embeddings: (1) code2vec (2) code2seq and (3) CuBERT

- a vector of source code metrics

- a vector of source code metrics and votes of heuristic-based detectors.

We include multiple heuristic-based detectors as our baselines.

Our target code smells are God Class and Long Method. We chose these code smells as they are highly prevalent (Palomba et al., 2018a) (i.e., developers frequently create this code smell while programming) and have a high negative impact on developer performance (Cairo et al., 2019), software quality attributes (Palomba et al., 2018a; Lacerda et al., 2020), and software reliability (Palomba et al., 2018a; Piotrowski \& Madeyski, 2020). They frequently co-occur with other code smells (Palomba et al., 2018b; de Paulo Sobrinho et al., 2018), which was proven to be especially problematic (Palomba et al., 2018a; Yamashita \& Moonen, 2013; de Paulo Sobrinho et al., 2018).

We consider the binary classification problem - we classify the code samples as smelly (positive class) or non-smelly (negative class). To fairly compare the performance of the algorithms, we compare all algorithms using the same experimental setting. In our experiments, the ML classifier trained using CUBERT source code embeddings achieved the best performance for both God Class (F-measure of 0.53) and Long Method detection (F-measure of 0.75 ). God Class detection proved to be a much more challenging task compared to the Long Method detection. The reason might lie in the inconsistency of class-level annotations in the used dataset. This inconsistency may be alleviated by applying welldefined guidelines for annotating a code smell, modeled after the Natural Language Processing field guidelines. Furthermore, as God Class is a class level smell and Long Method is a method level smell, the God Class detector must deal with a more significant input variability than the Long Method detector.

The contributions of this paper are as follows:

- We evaluated the effectiveness of three state-of-the-art neural source code embeddings: code2vec, code2seq, and CuBERT on the task of God Class and Long Method detection. This 
study is the first to evaluate the usefulness of pre-trained neural source code embeddings for code smell detection.

- This study is the first to perform code smell detection on the MLCQ dataset.

- This study is the first to compare the performances of multiple heuristic-based detectors on the same large-scale, manually labeled dataset.

- We provide a comprehensive replication package $^{1}$ researchers can use to replicate our experiments.

The rest of the paper is organized as follows. Section 2 defines our target code smells and explains the need for their detection and removal. In section 3, we explain the experiment we designed to compare different code smell detection approaches fairly. Sections 4 to 6 motivate our choice of code smell detection approaches and provide their application details. Section 7 provides and discusses the result of our experiment. Section 8 lists the threats to validity that may affect our conclusions. Finally, section 9 concludes our paper.

\section{Target code smells}

Many cataloged code smells exist (Sharma \& Spinellis, 2018), and addressing all smell types would be challenging due to their varied nature. Azeem et al. (2019) recommend that researchers consider the smell harmful when deciding which smells to target. Palomba et al. (2018a) warn that the smell prevalence should also be taken into account, for though a specific smell type may be considered harmful, it may not be as relevant if this type of smell rarely occurs in software projects. In this section, we define God Class and Long Method as our target code smells. Examining the available empirical studies argues that these smells have a high negative impact and that developers frequently create them while programming.

The God Class $^{2}$ code smell represents a class that implements many responsibilities and contains a large part of the system's logic (Sharma \& Spinellis, 2018). It disregards an essential principle of objectoriented programming that each component in the system should have one purpose and one responsibility. God classes are typically huge classes with many attributes and methods that are not interconnected, which means that the class has low cohesion ${ }^{3}$. Also, the God Class is generally associated with many other components in the system, which indicates high coupling.

The Long Method code smell occurs when a method is lengthy (Sharma \& Spinellis, 2018). Typically, these methods tend to centralize the functionality of the class. They are complex, challenging to understand and process large amounts of data from other classes. As God Classes, Long Methods represent a violation of the single responsibility principle of object-oriented programming.

God Class and Long method are both prevalent and harmful to software's quality attributes. Palomba et al. (2018a) conducted an extensive empirical investigation on code smells prevalence. They found that God Class and Long Method are among the seven most prevalent smells whose removal provides a high benefit in terms of change-proneness and thus should be removed to improve the overall maintainability of the code.

\footnotetext{
${ }^{1}$ https://github.com/Clean-CaDET/Y1-detectors-replication-package
}

2 In this paper we consider God Class, Large Class, Blob Class, and Brain Class as synonyms.

${ }^{3}$ Cohesion describes the extent to which the functions within a module are related. 
A recent tertiary systematic review (Lacerda et al., 2020) also concluded that the God Class and Long Method smells are amongst those that most affect different software quality attributes, God Class having the most considerable negative effect. Furthermore, instances suffering from these smells, besides negatively affecting quality attributes, are more bug-prone than others.

Another recent systematic literature review of papers that analyze the relationship of code smells and fault-proneness (Piotrowski \& Madeyski, 2020) has confirmed that code smells positively correlate with software defects. Thus, code smells are valuable predictors for fault detection models. However, not all types of smells are equally helpful - God Class and Long Method are exceptionally effective.

Another important finding is that the interaction of code smells can increase the change-proneness (Palomba et al., 2018a) and inhibit maintainability (Yamashita \& Moonen, 2013) of source code. The possible reason for this is that the co-occurrence of smells strongly hinders the developers' ability to understand the source code (Abbes et al., 2018). Alarmingly, Palomba et al. (2018b) found that smell cooccurrence is a widespread phenomenon. The same study found that God Class and Large class frequently co-occur with other smells.

\section{Experiment design}

To fairly compare the performance of our algorithms, we applied all code smell detection algorithms using the same experimental setting. In this section, we explain our experiment design. Section 3.1 explains why we chose the MLCQ dataset (Madeyski \& Lewowski, 2020) to train and evaluate our models. Then, in section 3.2, we describe the specifics of this dataset. Finally, in section 3.3, we explain our experiment design and chosen performance measure.

\subsection{Choosing the dataset}

A principal challenge for applying $\mathrm{ML}$ algorithms to the code smell detection problem is the need for a large, manually labeled dataset.

Manually labeling code smells in the source code is time-consuming (Azeem et al., 2019) and challenging (Hozano et al., 2018). To combat this issue, many researchers train their models on the datasets entirely built by applying heuristic-based tools (Azeem et al., 2019; Boutaib et al., 2021) or on synthetically generated data (Liu et al., 2019). However, we cannot reliably use such datasets to evaluate ML-based code smell detectors. The automatic annotation procedure does not guarantee that the dataset does not contain falsely labeled instances. Heuristic-based tools suffer from a high rate of false positives (software artifacts wrongly detected as being affected by a smell) (Sharma \& Spinellis, 2018).

Fontana et al. (2016) manually labeled instances from the Qualitas corpus for the presence of four code smells. In their annotation procedure, they used five heuristic-based detectors to identify a set of code smell candidates. Then, three MSc students manually examined and labeled code smell candidates, ensuring that one-third of the instances have positive labels (represent code smells) while the remaining instances are negative. In a later study, Di Nucci et al. (2018) argued that the artificially enforced ratio of code smells to non-smells might affect the conclusions obtained on the Qualitas corpus.

Palomba et al. (2015) built the Landfill code smell dataset by manually annotating 243 instances from 20 open-source Java projects. In their annotation procedure, one author manually examined the projects to identify code smell instances, and then the second author validated these candidates to discard false 
positives. Unfortunately, we could not download this dataset as the hyperlinks in the paper are no longer valid (Madeyski \& Lewowski, 2020).

In a later study, Palomba et al. (2018a) built a larger dataset by applying the heuristic-based tools to lower the number of manually validated code snippets and then manually filtering the false positives. The advantage of this dataset is its size and diversity - it encompasses 395 releases of 30 open-source systems. However, the study aimed not to train ML models for code smell detection but rather assess the diffuseness of code smells and their impact on code change- and fault-proneness. As such, this dataset has several limitations that limit its applicability for training ML models for code smell detection. Firstly, due to the employed semi-manual annotation procedure, there is no guarantee that this dataset does not contain false-negative instances. Secondly, it is unclear whether the annotators received training and guidelines for code smell detection. Finally, as Madeyski \& Lewowski (2020) pointed out, the dataset is not published in the form that can be used for reliable reproduction as it lacks information such as project URLs, commit hashtags, and full classpaths.

In this study, we use the MLCQ dataset (Madeyski \& Lewowski, 2020) as it is the largest dataset in which multiple annotators manually analyzed the smelliness of each code sample. As MLCQ authors stated, the advantage of this dataset over other existing code smell datasets is that it provides all information needed for reliable reproduction. In contrast to (Fontana et al., 2016), random sampling of code samples other datasets should result in a near-natural ratio of code smells and none-smells. In contrast to (Palomba et al., 2018a), each instance in the dataset was manually annotated. Finally, in contrast to all other datasets where the annotators were MSc students, the reviewers involved in the code smell assessment in the MLCQ dataset were active in the software development industry.

\subsection{MLCQ dataset}

Madeyski and Lewowski (2020) complied the MLCQ dataset through collaboration with 26 professional software developers. They gathered the code samples from 792 active industry-relevant, contemporary Java open-source projects and asked developers to review the collected code samples for bad smells. In this way, nearly 15000 code samples were annotated for four code smell types: God Class, Long Method, Feature Envy, and Data Class. Records in the MLCQ data set contain:

- The type of smell and its severity (none, minor, major, or critical)

- Crucial information for retrieval of the code sample and the software project that contains it: repository link, revision, and the exact location of the source code's smell (a particular method for the Long Method and Feature Envy smells, or a particular class for the God Class and Data Class code smells)

- The ID of the professional developer that provided the annotation.

We note that some of the code samples were annotated by multiple developers. Some annotations were cross-checked ${ }^{4}$, and that there is a non-negligible disagreement among the reviews. Annotator disagreement is more pronounced for class-level smells than for method-level smells (Madeyski \& Lewowski, 2020). Thus, there is some ambiguity of how to generate the ground-truth label for the smell. In our work, we use the majority-vote label for the samples where multiple conflicting annotations are available.

${ }^{4}$ Only samples tagged with a severity higher than "none" were considered for cross-checking. Samples selected for cross-checking were samples with a single review or samples with two conflicting annotations. 
Madeyski and Lewowski purposefully omitted annotator guidelines for recognizing particular code smells. They aimed to extract professional developers' contemporary understanding of code smells instead of imposing thresholds from the legacy literature. They asked the developers to answer an extensive survey on their professional experience in software development and code smells' perception. We use this information on the developer's background when analyzing our models' performance (chapter 7.3).

However, this annotation approach might prove problematic for training a ML model for code smell prediction. It is essential to create a domain-specific dataset with high-quality annotations to train and evaluate ML models of maximum accuracy in a particular domain. For example, in the NLP field, expertbased corpus acquisition has well-defined guidelines and best practices on creating consistent and highquality annotations. Annotating a dataset requires training the annotators and is referred to as "the science of annotation" (Hovy, 2010; Ide \& Pustejovsky, 2017).

It should be noted that we were not able to retrieve all code samples listed in the MLCQ dataset. As Madeyski and Lewowski (2020) pointed out, when a repository is removed, the project is no longer accessible. We include all available code samples in our analysis: 2408 unique class samples and 2255 unique method samples.

\subsection{Experimental setup}

We used the random stratified sampling procedure to divide the available code samples into the training (80\%) and test (20\%) set. The stratification procedure strived to produce an approximately equal ratio of all four severity levels in the training and test set. Table 1 presents the ratio of positive and negative samples in each part.

Table 1. The ratio of positive (code samples affected by the considered smell) and negative samples (code samples not affected by the considered smell) in train and test sets.

\begin{tabular}{l|llll}
\hline \hline MLCQ Dataset & \% smell instances & No. instances (pos/neg) & No. instances train (pos/neg) & No. instances test (pos/neg) \\
\hline Long Method & 13.0 & $277 / 2131$ & $223 / 1703$ & $54 / 428$ \\
God Class & 12.2 & $251 / 2049$ & $197 / 1642$ & $54 / 407$ \\
\hline \hline
\end{tabular}

We consider the binary classification problem - we classify the code samples as smelly ("minor," "major," or "critical" severity) or non-smelly (severity "none"). We consider the smelly class as the positive class and the non-smelly class as the negative class. This classification problem is highly imbalanced (Table 1), with the positive class being the minority class. Thus, as a performance measure, we calculate the F1-measure of the positive class:

$$
F 1=2 \cdot \frac{\text { precison } \cdot \text { recall }}{\text { precision }+ \text { recall }}
$$

where:

$$
\text { precision }=\frac{T P}{T P+F P}, \text { recall }=\frac{T P}{T P+F N},
$$

where $T P$ denotes the number of true positives, $F P$ denotes the number of false positives, $T N$ denotes the number of true negatives, and $F N$ denotes the number of false negatives. 
In our paper, we train ML classifiers using the multiple source code vector representations:

- three neural source code embeddings: (1) code2vec (2) code2seq and (3) CUBERT

- a vector of source code metrics

- a vector of source code metrics and votes of heuristic-based detectors

On each representation, we train the following ML classifiers: Random Forest, Bagging (using Support Vector Machines as the base algorithm), and Gradient Boosted Trees (XGBoost). We used the classifier implementations from the Scikit-learn (Pedregosa et al., 2011) and XGBoost (Chen \& Guestrin, 2016) libraries. We optimized the hyper-parameters of these models by performing a stratified 5 -fold-cross validation on the training set and using the Bayesian optimization strategy.

As the problem is highly imbalanced, we experimented with several sampling strategies from the imbalanced-learn package (Lemaître et al., 2017). Thus, we train each ML classifier on:

- the dataset resampled using the SMOTE (Synthetic Minority Over-sampling Technique) oversampling of the minority class,

- the dataset resampled using the Neighborhood Cleaning Rule undersampling to remove noisy samples from the dataset,

- the dataset resampled using a combined strategy of SMOTE minority oversampling and Edited Nearest Neighbor undersampling (SMOTEENN),

- the original dataset (without resampling).

When performing resampling, we made sure to resample only the training portion of the data and leave the validation data (for optimizing the model's hyper-parameters) and test data (for evaluating the model's performance) unchanged.

To ensure the reproducibility of our experiments, we fixed the random seed value in our experiments.

\section{Heuristic-based detection}

Most existing code smell detectors are heuristic-based (Azeem et al., 2019), and most heuristic-based approaches are metric-based (Bafandeh Mayvan et al., 2020). Metric-based approaches detect code smells by calculating a particular set of code metrics and applying predefined thresholds for each code metric. Although metric-based smell detection approaches have reasonable performance, several limitations hinder their adoption in practice.

The existing tools suffer from a high rate of false positives (software artifacts wrongly detected as being affected by a smell) (Sharma \& Spinellis, 2018). Many code smell candidates increase the needed human effort in fixing the code smells, as the developers need to analyze many code snippets to identify actual problems. The required effort may exceed the budget allocated for refactoring (Bafandeh Mayvan et al., 2020).

Metric-based approaches require the specification of thresholds for each metric. The selection of these thresholds greatly influences smell detectors' performance (Azeem et al., 2019), and there is no standardized way of choosing them.

The available approaches differ on the set of source code metrics they consider relevant for code smell detection. Additionally, even the approaches that use a similar set of metrics may differ in the 
predefined thresholds. Consequently, the agreement between different heuristic-based detectors is low (Azeem et al., 2019). There is currently no agreement on correct results and no standard criteria for comparing the detectors' performance (Bafandeh Mayvan et al., 2020).

The implementation of a metric-based approach may be precarious as the code metrics definitions lack standardization. For example, the Coupling Between Objects metric ( $C B O)$ metric is a long-established and widely used metric for code smell detection in object-oriented languages (Azeem et al., 2019) that measures the extent of coupling between two classes. However, the coupling can be measured and interpreted in many ways (Briand et al., 1999; Child et al., 2019). Consequently, such ambiguities in metric definitions led to different metrics tools producing widely inconsistent results even for wellknown metrics (Lincke et al., 2008; Sharma \& Spinellis, 2018).

Furthermore, the metric tools' documentations often quote a standard definition of the calculated metrics, with scant detail on how they handle ambiguities in their implementations (Child et al., 2019). Similarly, some studies proposing a metric-based smell detection approach lack precise definitions of the metrics they use. This lack of precision further complicates threshold specification as thresholds may depend on the tool used for metric value calculation.

To overcome the limitations of heuristic-based approaches, recently, the researchers started experimenting with ML-based techniques for smell detection. Azeem et al. (2019) conducted a systematic literature review on the proposed ML techniques for code smell detection and performed a meta-analysis reported performances. According to their meta-analysis, ML techniques generally outperform heuristic-based methods. However, there is no established benchmark for comparing different techniques, and some studies found that heuristic techniques perform slightly better than ML techniques (Pecorelli et al., 2020). Thus, when developing a ML-based approach for code smell detection, it is essential to include heuristic-based approaches as a baseline to show if and when ML is called for (Allamanis et al., 2018).

Recently, Bafandeh Mayvan et al. (2020) conducted a systematic literature review on bad smell specification and metric-based code smell detection approaches. To select the baseline metric-based detection approaches, we started from the list of metric-based rules for God Class and Long Method neatly summarized in their paper. We eliminated the rules for which we could not find precise metric definitions in the original paper that proposed the rule. For the remaining rules, we carefully examined the metric definitions provided in the documentation of the tools we used for metric extraction and eliminated the rules which use different definitions of the used metric or use the metrics the tools do not calculate. Table 2 shows the final set of rules we implemented as heuristic-based baselines. Tables 3 and 4 show the class-level and method-level metric definitions and the metric tools we used to calculate them.

When choosing the tools to extract metric values, we had to impose certain requirements. To cope with ambiguity in calculating the metrics, we need the tools to be open-source and well documented. As MLCQ encompasses many projects (792), we needed the tools to do batch computation and export data in a parseable format. We also looked for the tools that extract the methods through static analysis (i.e., they do not require compiled code) to avoid compilation issues. We selected the CK Tool (Aniche, 2015) and RepositoryMiner (Barbez et al., 2019) for metric extraction based on these requirements. 
In our experiments, we test the performance of the individual rules and their three aggregations. Let us denote the number of rules as $K$ and the probability of rule $k$ classifying the instance $x$ as suffering from a considered smell as $P\left(\hat{y}_{k}=1 \vee x\right)$ :

$$
P\left(\hat{y}_{k}=1 \mid x\right)=\left\{\begin{array}{ll}
1, & \text { if } \mathrm{x} \text { is a smell according to rule } k \\
0, & \text { if } \mathrm{x} \text { is not a smell according to rule } k
\end{array} .\right.
$$

We evaluate the following three models:

- $\quad$ ALL - the model predicts that the code sample suffers from the smell if all individual rules agree it suffers from the considered smell:

$$
P\left(\hat{y}_{A L L}=1 \mid x\right)=\bigwedge_{k=1}^{K} P\left(\hat{y}_{k}=1 \mid x\right)=1 .
$$

- ANY - the model predicts that the code sample suffers from the smell if ANY of the individual rules classify it as the considered smell:

$$
P\left(\hat{y}_{A N Y}=1 \mid x\right)=\bigvee_{k=1}^{K} P\left(\hat{y}_{k}=1 \mid x\right)=1 .
$$

- Weighted vote - the model calculates the probability of the code sample suffering from the smell as a weighted vote of the individual classifiers:

$$
P\left(\hat{y}_{\text {vote }}=1 \mid x\right)=\sum_{k=1}^{K} w_{k} \cdot P\left(\hat{y}_{k}=1 \mid x\right),
$$

where $w_{k}$ is the weight of the rule $k$. If $P\left(\hat{y}_{\text {vote }}=1 \mid x\right) \geq 0.5$, we classify the code sample as suffering from the considered smell; otherwise, we classify it as non-smell. We derive the weights of individual rules $w_{k}, k \in\{1, \ldots, K\}$ according to the $F$-measure the rules achieved on the training set. More precisely, we treat the $F$-measures of an individual rules as their scores $\left[s_{1}, s_{2}, \ldots, s_{K}\right]$. We then apply the softmax function to the rule score vector $\left[s_{1}, s_{2}, \ldots, s_{K}\right]$ to obtain the rule weights $\left[w_{1}, \ldots, w_{K}\right]$. 
Table 2. Heuristic-based approaches summarized in (Bafandeh Mayvan et al., 2020) we used as baselines in our experiments.

\begin{tabular}{|c|c|c|}
\hline Code smell & Denotement & Rule specification \\
\hline \multirow[t]{8}{*}{ God Class } & GC_1 & {$[$ ATFD $]>2 \&[$ WMC $] \geq 47 \&[$ TCC $]<0.33$} \\
\hline & GC_2 & {$[\mathrm{WMC}] \geq 47 \&[\mathrm{TCC}]<0.3 \&[\mathrm{ATFD}]>5$} \\
\hline & GC_3 & {$[\mathrm{NOM}]>15 \mid[\mathrm{NOF}]>15$} \\
\hline & GC_4 & {$[\mathrm{CLOC}]>750 \mid[\mathrm{NOM}]+[\mathrm{NOF}]>20$} \\
\hline & GC_5 & {$[\mathrm{NOM}]+[\mathrm{NOF}]>20$} \\
\hline & GC_6 & {$[\mathrm{LCOM}] \geq 0,725 \&[\mathrm{WMC}] \geq 34 \&[\mathrm{NOF}] \geq 8 \&[\mathrm{NOM}] \geq 14$} \\
\hline & GC_7 & {$[\mathrm{NOM}]>20|[\mathrm{NOF}]>9|[\mathrm{CLOC}]>750$} \\
\hline & GC_8 & {$[\mathrm{CLOC}]>100 \mid[\mathrm{VG}]>20$} \\
\hline \multirow{3}{*}{$\begin{array}{l}\text { Long } \\
\text { Method }\end{array}$} & LM_1 & $\mathrm{MLOC}>50$ \\
\hline & LM_2 & $M L O C>30 \& V G>4 \& N B D>3$ \\
\hline & LM_3 & $M L O C>50 \mid V G>10$ \\
\hline
\end{tabular}

Table 3. The definition of source code metrics used in heuristic-based approaches for God Class detection.

\begin{tabular}{|c|c|c|c|c|}
\hline Metric & Acronym & Definition & $\begin{array}{l}\text { Metric extraction } \\
\text { tool }\end{array}$ & $\begin{array}{l}\text { Metric acronym in tool } \\
\text { output }\end{array}$ \\
\hline Number of methods & NOM & Number of methods in a class & \multirow[b]{3}{*}{ CK Tool } & TotalMethodQty \\
\hline Number of fields & NOF & Number of attributes of a class & & TotalFieldsQty \\
\hline Tight class cohesion & TCC & $\begin{array}{l}\text { The relative number of method pairs of a } \\
\text { class that access in common at least one } \\
\text { attribute of the measured class }\end{array}$ & & tcc \\
\hline Lines of code & CLOC & Lines of code in a class & \multirow{4}{*}{ RepositoryMiner } & LOC \\
\hline Access to foreing data & ATFD & $\begin{array}{l}\text { The number of attributes from unrelated } \\
\text { classes accessed directly or by invoking } \\
\text { accessor methods }\end{array}$ & & ATFD \\
\hline Weighted method count & WMC & $\begin{array}{l}\text { The sum of the static complexity of all } \\
\text { methods in a class. The McCabe's } \\
\text { complexity (cyclomatic complexity) is } \\
\text { used to quantify the method's } \\
\text { complexity }\end{array}$ & & WMC \\
\hline Lack of cohesion & LCOM5 & $\begin{array}{l}\text { Provides a measure for the lack of } \\
\text { cohesion of a class. Lack of cohesion } \\
\text { between the methods of the class }\end{array}$ & & LCOM5 \\
\hline
\end{tabular}

Table 4. The definition of source code metrics used in heuristic-based approaches for Long Method detection.

\begin{tabular}{lllll}
\hline \hline Metric & Acronym & Definition & $\begin{array}{l}\text { Metric extraction } \\
\text { tool }\end{array}$ & $\begin{array}{l}\text { Metric acronym in tool } \\
\text { output }\end{array}$ \\
\hline Lines of code & MLOC & Lines of code in a method & loc \\
McCabe's complexity & VG & Cyclomatic complexity of the method & CK Tool & wmc \\
Nested blocks depth & NBD & Nested blocks depth & maxNestedBlocksQty \\
\hline
\end{tabular}




\section{Classifier trained on code metrics}

To train an ML model for code smell detection, we first need to represent the code snippets in our dataset as fixed-length vectors of real numbers. In most ML-based code smell detection approaches, researchers represent the code snippet by extracting features hand-engineered by the domain experts, i.e., the code metrics (Azeem et al., 2019). In this paper, we consider this approach of training ML models using hand-crafted features as a baseline method against which we compare the performance of ML models trained on automatically inferred features (section 6).

An advantage of using hand-crafted features is that they allow us to analyze and interpret the behavior of $\mathrm{ML}$ models trained using them. We rely on these features when performing ML model error analysis (section 7.3). A disadvantage of this approach is that designing helpful features is challenging. It requires domain expertise, is time-consuming, and is problem-dependent: features engineered for a particular software engineering task might not be helpful in a different context (Wang et al., 2020).

An alternative to hand-crafting features is to automatically infer helpful features through deep learning (section 6). However, this approach may lead to a slight performance improvement (Rabin et al., 2020) while sacrificing model interpretability. Thus, it is vital to include models trained on manually engineered features as baselines to estimate if the performance improvement justifies the added model complexity (Allamanis et al., 2018).

In our experiment, we train ML models on code metrics extracted using the CK Tool and RepositoryMiner metric extraction tools we used to facilitate the implementation of heuristic-based detectors. We include all extracted metrics, regardless of whether we use them in heuristic baselines or not. In cases where both tools calculate the same metric, we choose the metric whose implementation corresponds to the metric definition used in heuristic-based detectors. This way, we use a total of 46 class-level metrics for God Class detection and 26 method-level metrics for Long Method detection ${ }^{5}$. Before applying ML models, we performed the following preprocessing techniques:

- We encoded the categorical variables using label encoding.

- We used -1 to denote that the value of a particular metric cannot be calculated for the analyzed code sample.

- We normalized the metric values using z-normalization.

\section{Classifier trained on neural source code embeddings}

The previous section discussed how the analyzed source code snippets could be represented as a vector of hand-crafted features to apply ML models. An alternative way of representing source code as a fixedlength vector of real numbers is through neural source code embeddings (Alon et al., 2019). In this approach, embeddings are learned by training deep neural networks to do a proxy task (e.g., method name prediction), for which labeled training data is plentiful. The goal is that the learned representation of the source code should preserve its semantic meaning - semantically similar source code snippets should be mapped to similar vectors (Alon et al., 2019).

This large-scale pretraining of code embeddings is beneficial for ML applications in which labeled data is scarce (Hussain et al., 2020). One such application is code smell detection, as manual labeling of code

\footnotetext{
${ }^{5}$ The exact metrics and their extracted values can be found in our replication package https://github.com/Clean-
} CaDET/Y1-detectors-replication-package. 
smells is time-consuming (Azeem et al., 2019) and challenging (Hozano et al., 2018). Fortunately, if we use the embeddings trained to capture code's semantics, we might require far less training data than the amount necessary to train a code smell detection model from scratch.

Researchers proposed many source code embeddings. In section 6.1, we motivate our choice to use three representative embeddings for our code smell detection task: code2vec, code2seq, and CuBERT. Sections 6.2 to 6.4 explain how we use the chosen embeddings to obtain methods' representations. Finally, section 6.5 explains how we obtain the embedding of classes.

\subsection{Applying source code embeddings in Software Engineering tasks}

The current practices of developing source code models can be divided into three groups: representations that capture the structural nature of code, NLP-inspired models, and code change models.

Structural embeddings model the codes' AST (Neamtiu et al., 2005; Alon et al., 2019; Alon et al., 2018), data flow (Ben-Nun et al., 2018), or control flow graph (DeFreez et al., 2018). Well-known embeddings from this group that the community extensively adopted due to their public tool support (Pour et al., 2021) are code2vec (Alon et al., 2019) and code2seq (Alon et al., 2018). These embeddings were used in various software engineering tasks such as code comment generation, code authorship identification, code clones detection (Kang et al., 2019), code summarization (Wang et al., 2021), detection of source code vulnerabilities (Coimbra et al., 2021), and semantic code search (Arumugam, 2020). Embeddings that capture the structural nature of code might be beneficial for God Class and Long Method detection. Code snippets that suffer from these smells have high structural complexity that hinders the codes' readability (Luburić et al., 2021). Our study is the first to use these embeddings for code smell detection to the best of our knowledge.

NLP contextual embeddings capture the text semantics (Bakarov, 2018). Code embeddings inspired by these techniques hold the potential to capture source code semantics crucial for many software engineering tasks such as software defect prediction (Pan et al., 2021), automated program repair (Mashhadi \& Hemmati, 2021), and vulnerability detection (Wu, 2021). Karampatsis and Sutton (2020) used the ELMo framework (Peters et al., 2018) to train their embeddings and achieved state-of-the-art results for bug detection. CodeBERT (Feng et al., 2020), based on BERT (Devlin et al., 2018), achieved state-of-the-art results on natural language code search and code documentation generation tasks. Recently, Kanade et al. (2020) proposed CUBERT, a code-understanding BERT model, that outperformed other embeddings on multiple software engineering benchmark tasks. As the codes' semantics are crucial for God Class and Long Method detection (Luburić et al., 2021), we apply CuBERT for this task.

Embeddings that model code changes encode the difference in the syntactic structure of code before and after a change. Such embeddings are helpful for the classification of security-relevant commits (Sabetta \& Bezzi, 2018; Lozoya et al., 2021), log message generation, bug fixing patch identification, and just-in-time defect prediction (Hoang et al., 2020). We did not consider this group of embeddings for code smell detection, although code change history can be helpful for God Class detection (Barbez et al., 2019; Palomba et al., 2014). The MLCQ dataset contains samples from 792 projects and extracting the needed embeddings would be extremely time intensive. Thus, we leave this idea for future research. 


\section{2 code2vec}

Alon et al. (2019) proposed code2vec, a deep neural network that learns code embeddings. Their neural network model input is the vector representation of the code snippet, and the output is a corresponding tag. For example, Alon et al. used the method's body as the input code snippet and the methods' name as the output tag.

To obtain the models' input (source code snippets' vector representation), Alon et al. propose representing the code snippet as an unordered set of paths in its AST:

1. A code snipped is parsed to produce its AST.

2. The paths between all pairs of AST leaves are extracted. A single path is a tuple of the two leaf nodes in AST and the path (an ordered sequence of AST nodes) between them.

3. Each path is assigned a weight that corresponds to its' importance to the models' output. An attention mechanism is used to learn the paths' importance.

4. A single vector representation of the code snippet is calculated as a weighted average of the path vectors.

We used code2vec to represent each method in our dataset as a fixed-length vector for our code smell detection task. We used the implementation provided by Alon et al. (2019):

1. We construct the methods' AST paths using the path extractor for the Java programming language.

2. We input the extracted AST paths to the Alon et al. trained open-sourced Java model. We remove the last softmax layer from the model and use the feature vector ("code vector") as the models' output. We treat this 384-dimensional vector as a representation of the method.

\section{3 code2seq}

Code2Seq (Alon et al., 2018) is an encoder-decoder model that learns the vector representation of the source code. Same as code2vec, the models' input is the vector representation of the code snippet, and the output is a corresponding natural language sequence (e.g., the method name).

Like code2vec, the models' input is constructed from the code snippets' AST:

1. A code snippet is parsed to produce its AST.

2. The paths between all pairs of AST leaves are extracted. A single path is an ordered sequence of AST nodes.

3. A bi-directional LSTM encodes each AST path as a fixed-length sequence of nodes.

4. The decoder uses attention to select relevant paths while decoding to generate an output sequence.

We used code2seq to represent each method in our dataset as a 320-dimensional vector for our code smell detection task. We used the implementation provided by Alon et al. (2018) who provided the extractors for preprocessing source code of the input methods and the open-sourced model for Java programming language.

\subsection{CUBERT}

The transformer model and context-dependent embeddings have revolutionized the field of NLP, with the BERT model (Devlin et al., 2018) as one of the first and most prominent examples. Having that in 
mind, Kanade et al. (2020) built the CUBERT model. CuBERT is essentially the "Large" variant of the BERT model pre-trained on code snippets instead of natural language. BERT Large is a transformer (Vaswani et al., 2017) with 4 layers with 16 attention heads that provides a 1024-dimensional vector as embedding for each token. Originally Kanade et al. (2020) trained CUBERT on Python code but have subsequently published a version pre-trained on Java code snippets which we used in our experiments.

As input to the BERT Large model is a sequence of tokens, we preprocessed the Java code snippets using a custom Java tokenizer developed by Kanade et al. Then, we input each sequence (line of Java code) into the CUBERT model. We then extracted the 1024-dimensional embedding from a special start-ofexample ([CLS]) token to represent each line of code, as recommended by the original BERT authors (Devlin et al., 2018).

\subsection{Embedding methods and classes}

Long Method is a method-level smell. Pre-trained code2vec and code2seq models expect the methods' body as an input code snippet. Thus, we use the methods' body as input to obtain a method-level embedding and consider the resulting embedding vector as the methods' vector representation.

God Class is a class-level smell. Pre-trained code2vec and code2seq models cannot directly embed a class. Thus, to perform predictions at the class level, we need to define the appropriate class embedding. Compton et al. (2020) addressed this problem by treating classes as collections of methods. They evaluated the efficacy of class embeddings that constitute simple mathematical operations on sets of method embeddings. In their experiment, the mean aggregation method achieved the best performance. Thus, we adopted this approach for embedding classes using code2vec and code2seq models: to embed a class, we embed all its constituting methods and calculate the mean of the resulting code embedding vectors.

The pre-trained CUBERT model expects a single line of code as an input. Thus, to embed a method using the CUBERT model, we embed the method by embedding each line of code in a methods' body and then summing all resulting vectors. Similarly, to embed the class, we embed all its constituting methods and calculate the sum of the resulting code embedding vectors. Like Compton et al. (2020), we evaluated the efficacy of different mathematical operations on sets of method embeddings (mean, concatenation, and summation) and concluded that summation resulted in the highest performance for this task.

\section{Results}

This section presents the results of our experiment. In section 7.1, we evaluate the heuristic-based code smell detectors on the training set and select the best-performing heuristic. In section 7.2, we compare this best-performing heuristic to ML-based approaches on the test set. Finally, in section 7.3, we perform the error analysis for our best-performing approach.

\subsection{Heuristic-based detection}

Table 5 presents the performance of heuristic-based rules on the task of God Class detection, and Table 6 presents the performance of heuristic-based rules on the task of Long Method detection.

As the application of heuristic-based detectors does not require training, we report the performance of heuristic-based approaches on the training set, the test set, and the whole dataset (training and test set combined). Note that, in a realistic scenario, we would need to choose the best-performing algorithm 
using the training data exclusively. Thus, the best performing individual rule for God Class detection is GC_8 (Table 5), and the best performing individual rule for Long Method detection is ANY (Table 6).

For the God Class smell (Table 5), our aggregations of the individual rules could not improve the performance compared to the individual rule performance. As expected, the ALL approach has the best precision of the compared heuristic-based approaches, but its recall is extremely low. In contrast, ANY approach has low precision but the highest recall. The Weighted Vote approach has the same performance as the rule GC_8. The GC_8 vote dominates the Weighted Vote as this rule achieves a significantly higher F-measure on the training set than other rules. As GC_8 is a more simplistic approach than Weighted Vote and achieves the same performance, we consider GC_ 8 the best performing heuristic for God Class detection in our experiment. In Table 6, we observe the same trends for our aggregation approaches for the Long Method smell. However, the ANY model has a significantly higher F-measure on the training set than other approaches. We consider the ANY approach as the best performing heuristic for Long Method detection in our experiment.

A surprising result for the God Class smell (Table 5) is that, although the ALL approach has the highest precision of all heuristic-based strategies, its precision on the whole dataset is still relatively low (58\%). We would expect ALL to have near-perfect precision as, for the samples it labels as God Class, there is a perfect agreement of diverse God Class detection rules. For comparison, the ALL approach for Long Method has an extremely high precision of $97 \%$, as expected. To understand the modest precision of ALL approach for God Class detection, we asked the domain expert to analyze classes mislabeled as suffering from the God Class code smell. The ALL approach labeled 12 classes as the God Class smell, and five of these classes are labeled as non-smell by the annotator majority vote in the MLCQ dataset. Our domain expert inferred that these five examples are mislabeled in the MLCQ dataset - he would label these examples as major to critical. To further investigate this issue, we looked at individual annotations in the MLCQ dataset and the annotator experience ${ }^{6}$. We found that three out of these five annotations had low annotator agreement. It is interesting to observe that the MLCQ annotator with a lot of professional software engineering experience and the largest number of annotated samples in the MLCQ dataset labeled these examples as "critical." The two remaining code samples were labeled as "none" by a single annotator and were not cross-checked. This finding indicates that the MLCQ dataset has shortcomings that might affect the training and evaluation of the ML-based God Class detectors. A more systematic approach to code smell labeling might be required to overcome this issue.

Unfortunately, it is hard to compare the results reported in tables 5 and 6 to the results reported in the existing literature. Many studies that proposed the considered rules use case studies as the evaluation method (Rasool \& Arshad, 2015). Typically, in these case studies, the rules are applied to a small number of projects, and the authors qualitatively evaluate the results without reporting the aggregate measures such as accuracy. The studies that do report the aggregate measures report either just accuracy, precision or recall. None of these studies reported the F-measure for the minority (smell) class to the best of our knowledge. Furthermore, we did not find a study that compares performances of these rules

\footnotetext{
${ }^{6}$ We derive the annotator experience from the accompanying annotator survey. We considered both the years of experience in software development and the number of provided annotations as important experience factors. Software engineering experience contributes to the quality of code smell detection. Providing many annotations indicates that the annotator has developed a consistent strategy for labeling code smells in diverse projects (Luburić et al., 2021).
} 
on the same large-scale, manually labeled dataset. Thus, we consider the results reported in tables 5 and 6 as one of the significant contributions of this study.

Table 5. Performance of heuristic-based approaches for God Class detection. We report the performance on the training set, the test set, and the whole dataset (All). We test the performance of both individual rules (G_1 to G_8) and three rule aggregations (ALL, ANY, Weighted Vote). The performance is reported in terms of precision ( $P)$, recall $(R)$, and F-measure ( $F$ ) of the positive (smell) class, which is the minority class in the dataset.

\begin{tabular}{|c|c|c|c|c|c|c|c|c|c|c|}
\hline & \multirow[t]{2}{*}{ Rule specification } & \multicolumn{3}{|c|}{ Training set } & \multicolumn{3}{|c|}{ Test set } & \multicolumn{3}{|l|}{ All } \\
\hline & & $\mathbf{P}$ & $\mathbf{R}$ & $\mathbf{F}$ & $\mathbf{P}$ & $\mathbf{R}$ & $\mathbf{F}$ & $\mathbf{P}$ & $\mathbf{R}$ & $\mathbf{F}$ \\
\hline GC_1 & {$[$ ATFD $]>2 \&[\mathrm{WMC}] \geq 47 \&[\mathrm{TCC}]<0.33$} & 0.26 & 0.03 & 0.05 & 0.75 & 0.06 & 0.10 & 0.35 & 0.03 & 0.06 \\
\hline GC_2 & {$[\mathrm{WMC}] \geq 47 \&[\mathrm{TCC}]<0.3 \&[$ ATFD $]>5$} & 0.42 & 0.02 & 0.05 & 0.75 & 0.06 & 0.10 & 0.50 & 0.03 & 0.06 \\
\hline GC_3 & {$[\mathrm{NOM}]>15 \mid[\mathrm{NOF}]>15$} & 0.33 & 0.42 & 0.37 & 0.44 & 0.52 & 0.47 & 0.35 & 0.44 & 0.39 \\
\hline GC_4 & {$[\mathrm{CLOC}]>750 \mid[\mathrm{NOM}]+[\mathrm{NOF}]>20$} & 0.35 & 0.46 & 0.40 & 0.39 & 0.44 & 0.42 & 0.36 & 0.46 & 0.40 \\
\hline GC_5 & {$[\mathrm{NOM}]+[\mathrm{NOF}]>20$} & 0.35 & 0.46 & 0.40 & 0.39 & 0.44 & 0.42 & 0.36 & 0.46 & 0.40 \\
\hline GC_6 & $\begin{array}{l}{[\mathrm{LCOM}] \geq 0.725 \&[\mathrm{WMC}] \geq 34 \&[\mathrm{NOF}] \geq 8 \&} \\
{[\mathrm{NOM}] \geq 14}\end{array}$ & 0.31 & 0.56 & 0.40 & 0.34 & 0.61 & 0.43 & 0.31 & 0.57 & 0.40 \\
\hline GC_7 & {$[\mathrm{NOM}]>20|[\mathrm{NOF}]>9|[\mathrm{CLOC}]>750$} & 0.36 & 0.43 & 0.39 & 0.39 & 0.39 & 0.39 & 0.37 & 0.42 & 0.39 \\
\hline GC_8 & {$[\mathrm{CLOC}]>100 \mid[\mathrm{VG}]>20$} & 0.34 & 0.66 & 0.45 & 0.37 & 0.70 & 0.49 & 0.35 & 0.67 & 0.46 \\
\hline \multirow{3}{*}{$\begin{array}{l}\text { Rule } \\
\text { agg. }\end{array}$} & ALL & 0.50 & 0.02 & 0.04 & 0.75 & 0.06 & 0.10 & 0.58 & 0.03 & 0.05 \\
\hline & ANY & 0.30 & 0.70 & 0.42 & 0.31 & 0.70 & 0.43 & 0.30 & 0.70 & 0.42 \\
\hline & Weighted Vote & 0.34 & 0.66 & 0.45 & 0.37 & 0.70 & 0.49 & 0.35 & 0.67 & 0.46 \\
\hline
\end{tabular}

Table 6. Performance of heuristic-based approaches for Long Method detection. We report the performance on the training set, the test set, and the whole dataset (All). We test the performance of both individual rules (LM_1 to LM_8) and three rule aggregations (ALL, ANY, Weighted Vote). The performance is reported in terms of precision $(P)$, recall $(R)$, and F-measure (F) of the positive (smell) class, which is the minority class in the dataset.

\begin{tabular}{|c|c|c|c|c|c|c|c|c|c|c|}
\hline & \multirow[t]{2}{*}{ Rule specification } & \multicolumn{3}{|c|}{ Training set } & \multicolumn{3}{|c|}{ Test set } & \multicolumn{3}{|l|}{ All } \\
\hline & & $\mathbf{P}$ & $\mathbf{R}$ & $\mathbf{F}$ & $\mathbf{P}$ & $\mathbf{R}$ & $\mathbf{F}$ & $\mathbf{P}$ & $\mathbf{R}$ & $\mathbf{F}$ \\
\hline LM_1 & MLOC > 50 & 0.93 & 0.26 & 0.40 & 1.00 & 0.20 & 0.34 & 0.94 & 0.24 & 0.39 \\
\hline LM_2 & $M L O C>30 \& V G>4 \& N B D>3$ & 0.88 & 0.24 & 0.37 & 0.91 & 0.18 & 0.31 & 0.89 & 0.23 & 0.36 \\
\hline LM_3 & MLOC > $50 \mid$ VG > 10 & 0.86 & 0.40 & 0.55 & 0.89 & 0.31 & 0.46 & 0.87 & 0.38 & 0.53 \\
\hline \multirow{3}{*}{$\begin{array}{l}\text { Rule } \\
\text { agg. }\end{array}$} & ALL & 0.96 & 0.11 & 0.20 & 1.00 & 0.11 & 0.20 & 0.97 & 0.11 & 0.20 \\
\hline & ANY & 0.85 & 0.47 & 0.61 & 0.86 & 0.33 & 0.48 & 0.85 & 0.44 & 0.58 \\
\hline & Weighted Vote & 0.86 & 0.40 & 0.55 & 0.89 & 0.31 & 0.46 & 0.87 & 0.38 & 0.53 \\
\hline
\end{tabular}

\subsection{Comparing the performance of different approaches for code smell detection}

This section compares the performance of different approaches for code smell detection we considered in this study. Tables 7 and 8 summarize the performances of different approaches measured on the test set for God Class and Long Method, respectively: the first column lists how we denote each considered code smell detection approach; the second column lists the used source code representation; the third 
column presents the used algorithm; the last three columns report the precision, recall, and F-measure of the minority (smell) class measured on the test.

Table 7. Performance of different approaches for God Class detection. We report the precision, recall, and f-measure of the minority (god class) class evaluated on the test set.

\begin{tabular}{l|l|l|lcc}
\hline \hline Denotement & Features & Approach & Precision & Recall & F-measure \\
\hline ML_code2vec & code2vec features & Random Forest+ SMOTEENN & 0.16 & 0.69 & 0.26 \\
ML_code2seq & code2seq features & Bagging (SVM classifier) + SMOTE & 0.34 & 0.50 & 0.41 \\
H_metrics & Code metrics & Heuristic detector (GC_8) & 0.37 & 0.70 & 0.49 \\
ML_metrics & Code metrics & Random Forest + SMOTEENN & 0.41 & 0.67 & 0.51 \\
ML_metrics\&votes & Code metrics + votes of heuristic & Random Forest + SMOTEENN & 0.43 & 0.69 & 0.52 \\
& detectors (GC_3 -GC_8) & & & 0.58 & 0.53 \\
ML_CuBERT & CuBERT features & Bagging (SVM classifier) + SMOTEENN & 0.48 & \multicolumn{2}{c}{0.58} \\
\hline \hline
\end{tabular}

Table 8. Performance of different approaches for Long Method detection. We report the precision, recall, and $f$-measure of the minority (long method) class evaluated on the test set.

\begin{tabular}{l|l|l|lcc}
\hline \hline Denotement & Used features & Approach & Precision & Recall & F-measure \\
\hline ML_code2vec & code2vec features & Random Forest + SMOTE & 0.33 & 0.19 & 0.24 \\
H_metrics & Code metrics & Heuristic-based approach (ANY) & 0.86 & 0.33 & 0.48 \\
ML_code2seq & code2seq features & Bagging (SVM classifier) + SMOTE & 0.50 & 0.60 & 0.55 \\
ML_metrics\&votes & Code metrics + votes of heuristic & Random Forest + SMOTEENN & 0.63 & 0.63 & 0.63 \\
ML_metrics & detectors (LM_1-LM_3) & Random Forest + SMOTEEENN & 0.63 & 0.70 & 0.67 \\
ML_CuBERT & Code metrics & XGBoost + SMOTEENN & 0.70 & 0.81 & $\mathbf{0 . 7 5}$ \\
\hline \hline
\end{tabular}

For each approach, we evaluate only the best-performing model. For the heuristic-based models, the best performing models are GC_8 for God Class detection and ANY for Long Method detection (section 7.1). For ML-based models, we select the best-performing model after hyperparameter tuning, according to the F-measure of the minority class evaluated through a 5 -fold-cross validation procedure on the training dataset.

From Tables 7 and 8, we can see that, in our experiment, the best performing approach for both God Class and Long Method detection proved to be a classifier trained using CUBERT source code embeddings as code snippet representation, ML_CUBERT. The next two best-performing approaches are the $\mathrm{ML}$ classifier trained using source code metrics $\mathrm{ML}$ metrics and the ML classifier trained using the combination of code metrics and votes of heuristic-based detectors as features ML_metrics\&votes.

Excluding the $M L$ classifier trained using the code2vec ( $\left.M L \_c o d e 2 v e c\right)$ and $M L$ classifier trained using code2seq features ( $M L \_$code2seq), ML classifiers outperformed the heuristic-based approaches in our experiments. This result contradicts the study (Pecorelli et al., 2020) that found that heuristic techniques perform slightly better than ML techniques but agrees with Azeem et al.'s (2019) meta-analysis. As discussed in section 4, applying heuristic-based approaches is brittle due to the ambiguity of code

\footnotetext{
${ }^{7}$ We excluded the votes of GC_1 and GC_2 as they achieved extremely low F-measure on the training set.
} 
metrics definitions and possible differences from the implementations of the same metric in different code metrics extraction tools. The significant advantage of ML-based detectors is that we can use the source code metrics as indicators, rather than imposing hardcoded thresholds that might need readjusting depending on the tool used for calculating code metrics.

Combining the heuristic-based detectors votes and code metrics for representing the analyzed source code (ML_metrics\&votes) did not improve the performance compared to representing the source code using code metrics alone (ML_metrics). The improvement is slight for the God Class detection ( $1 \%$ improvement), while it hinders the performance for the Long Method detection ( $4 \%$ decrease).

$M L$ code2vec achieved the worst performance on both tasks. We attribute this to the low generalizability of code2vec embeddings. Code2vec is a representative state-of-the-art among embedding models (Kang et al., 2019), and it has excellent performance on the method name prediction task Alon et al. (2019) designed it for. However, the learned code embeddings may not be generalizable to other tasks. Kang et al. (2019) investigated the generalizability of code2vec embeddings to three different software engineering tasks: code comment generation, code authorship identification, and code clones detection. They have demonstrated that code2vec's token embeddings do not always significantly increase models' performance for these tasks and cannot be used readily to improve simpler models.

Code2seq is an improvement of code2vec, proposed by Alon et al. (2018). In our experiments, $M L \_c o d e 2 s e q$ outperformed $M L \_c o d e 2 v e c$ on both tasks. However, ML_metrics based on the handcrafted features outperformed both embeddings. We may attribute this to the fact that these embeddings predominantly focus on the syntactic and lexical properties of the code with little to no semantic implication (Wang et al., 2021). On the other hand, CUBERT embeddings seem to capture the semantics needed for code smell detection.

In the case of God Class detection, the performance of $M L_{-}$CUBERT is not significantly better than classifiers trained using source code metrics $M L \_$metrics (2\% improvement) or their combination with votes of heuristic-based detectors as features $M L \_$metrics\&votes ( $1 \%$ improvement). However, ML_CUBERT performs significantly better on the Long Method detection task than $M L \_$metrics ( $8 \%$ improvement) and $M L \_$metrics\&votes ( $12 \%$ improvement).

We observe a similar trend when comparing $M L \_C U B E R T$ to the heuristic-based approach $H_{-}$metrics: the improvement is slight for the God Class detection (4\% increase) but substantial for the Long Method detection (27\% increase). Note that $H_{-}$metrics and $M L \_$metrics both rely on hand-crafted source code metrics.

This result indicates that the CUBERT features provide better source code representation for code smell detection than source code metrics and have the additional benefit of being automatically inferred. However, as the performance improvement for the God Class detection task is slight, we provide a deeper analysis of whether CUBERT features provide a better semantic representation of the analyzed source code than the source code metric in section 7.3.

According to the performance achieved by all tested approaches on the MLCQ dataset, God Class detection poses a much more challenging task compared to the Long Method detection. We may attribute this to one of the following reasons: 
- In the MLCQ dataset, the annotators had a significantly higher disagreement for class-level smells than for method-level smells (Madeyski \& Lewowski, 2020). Thus, for tackling the God Class detection problem successfully, a more systematic approach for labeling code smells that would produce higher-quality labels is needed. As pointed in section 3.2, the NLP field has welldefined guidelines and best practices for creating consistent and high-quality annotations. Unfortunately, to the best of our knowledge, current publicly available manually labeled datasets for code smell detection were not subjected to the same stringent annotation guidelines.

- The reason for the worse performance of God Class detectors may be due to God Class being a class level smell, while the Long Method is a method level smell. Therefore, the God Class detector must deal with a more significant input variability than the Long Method detector, and more labeled data is needed to tackle this smell.

\subsection{Performance analysis}

This section presents the error analysis we performed to analyze the reasons for different performances of CUBERT automatically inferred features and hand-crafted code metrics features. We applied ML_CUBERT, ML_metrics, and H_metrics to the examples in the test set and collected all examples where these approaches made a wrong decision. Then, we asked the domain expert to analyze these code samples. Our goal is to manually identify code characteristics that influence the performance of these models. In the remainder of this section, we list our conclusions.

The performance improvement for God Class detection was slight, but our error analysis indicates CUBERT features can capture the semantics needed to detect code smells. ML_CUBERT correctly labeled God Classes that have multiple responsibilities that metric-based approaches mislabeled. To ensure that the metrics-based approaches detect these samples, we need to develop metrics that measure the number of responsibilities and can be automatically calculated, which is challenging. In contrast to ML_metrics, ML_CUBERT also correctly labeled atypical classes. For example:

- classes with many auto-generated comments;

- classes that have many methods and many fields but semantically do not suffer from the God Class smell as they are relatively simple and do not handle multiple responsibilities;

- classes that follow design patterns ${ }^{8}$;

- classes that have a single complex method but are simple otherwise.

On the other hand, we concluded that ML_CUBERT might benefit from including the RFC metrics in the source code representation as it mislabeled several classes with large RFC that $M L$ metric correctly detected.

The performance improvement of ML_CUBERT over ML_metrics for the Long Method code smell was substantial, and we would need to design additional metrics to improve the performance of $M L \_m e t r i c s$. Designing new code metrics is a tedious task that requires domain expertise and is problem-specific. This result highlights the benefit of using the CUBERT approach of automatically inferring features over hand-crafting features for different code smell detection tasks.

\footnotetext{
${ }^{8}$ Design patterns typically violate clean code principles but would not be labeled as smells.
} 
A disadvantage of using code metrics as features is that this approach relies upon non-trivial metric extraction implementations. As discussed in section 4, metric extraction is complex, and metric implementation may not necessarily follow the metrics designer's reasoning and intention as metric definitions are often incomplete (Lincke et al., 2008). For example, it is ambiguous should the tool consider the inner classes when calculating the metrics of the outer class. If the inner classes should be considered, this adds to the complexity of the metric calculations. The implication is that the thresholds used in metric-based heuristics are tool-dependent and therefore brittle.

Moreover, the metric calculation might depend on including or excluding library classes, and calculating metrics for a particular code snippet might require parsing the whole project. For example, it was very time-consuming to extract the metrics needed to represent the code samples from the MLCQ dataset. MLCQ includes roughly around six samples per project. Regardless, we needed to examine the whole project to calculate some code metrics ${ }^{9}$ accurately. Furthermore, we needed to include library classes, which was impossible for some projects due to parsing errors. Thus, some metrics may not have been accurately calculated for some code samples, affecting the metric-based detectors.

For example, in contrast to $H_{-}$metrics and ML_metrics, $M L \_C U B E R T$ correctly detects Long Methods characterized by too many words and wide expressions. While $H_{-}$metrics does not rely on the number of unique words, $M L \_$metrics used this metric as one of the predictor variables. However, our domain expert recognized that this quantity was miscalculated (had a low value) for a part of these samples.

Consequently, some metrics may not have been accurately calculated for some code samples, affecting the metric-based detectors. In contrast, CUBERT embeddings require only the analyzed code samples as input. Thus, calculating the CUBERT embedding is more straightforward and less bug-prone.

We identified a need for a systematic approach to code smell annotations. For example, there is no consensus on whether inner classes should be included in the outer class analysis. It is unclear how the MLCQ annotators handled this issue. Additionally, during error analysis, our domain expert stated some potentially relevant guidelines for annotating code smells. For example, if the classes' complexity stems from a single Long Method, he would not label this class as a God Class. However, if the class has several Long Methods, he would label it as a God Class. Another guideline example would be to treat classes that follow design patterns differently when deciding on their smelliness. Developing a consensus on such guidelines, as is standard in the NLP field, would greatly improve the quality of the code smell datasets and consequently the state-of-the-art in this field.

Another related issue is that not all dataset examples are cross-checked and that there is a significant disagreement between the annotators, especially for the class-level smells. Additionally, our domain expert disagreed with the labels assigned to some of the code samples. These issues further indicate that we need more studies to derive a "science of annotation" in the code annotation field.

To summarize, we conclude that in our experiment, the best source code representation for code smell detection was CUBERT features for the following reasons:

\footnotetext{
${ }^{9}$ The examples of metrics that cannot be calculated accurately by examining the code snippet alone but require examination of other related code snippets are DIT (Depth of Inheritance Tree), CBO (Coupling Between Objects), and RFC (Response for a Class).
} 
- CUBERT features better captured the semantics needed for God Class and Long Method detection than code metrics

- Metrics extraction implementations are non-trivial as metric definitions can be ambiguous

- Metric extraction is time-consuming

- Metrics values may be miscalculated, which is hard to detect.

\section{Threats to validity}

Here we discuss the factors that might affect our conclusions: internal, external, and construct validity.

\subsection{Threats to internal validity}

Threats to internal validity are related to the correctness of our experimental results.

MLCQ dataset was labeled by 26 professional software developers of different backgrounds. However, not all instances were annotated by multiple developers, and, therefore, some assigned labels may be subjective. Another factor that might affect our experimental conclusions is the label aggregation strategy we used for the instances in the MLCQ dataset that had multiple labels assigned by different annotators. In such cases, we opted to derive the ground truth for each instance by the majority vote, which is a widely accepted way to assign a label in these situations (Ide \& Pustejovsky, 2017).

Finally, a single domain expert performed the error analysis, and therefore, our error analysis conclusions are susceptible to subjectivity.

\subsection{Threats to external validity}

Threats to external validity regard the generalizability of our experimental results. We used the MLCQ dataset that encompasses many active industry-relevant, contemporary projects. These projects are of varying sizes and encompass different domains. These factors mitigate the issue of generalizability. However, all projects are open-source and written in Java programming language, and we cannot guarantee that our conclusions generalize to other industry projects written in other programming languages.

\subsection{Threats to construct validity}

Construct validity concerns the relationship between the theory and empirical observations.

In our experiments, we used multiple metric-based heuristics to show the effectiveness of the ML approach. As discussed in section 4, applying metric-based heuristics is brittle due to possible differences between metrics definitions and their implementations. Threshold values defined by the heuristic authors might be inadequate if the metric definition differs from its implementation, consequently affecting the measured performance of heuristics. We mitigated this threat by choosing metric extraction tools that are open-source and documented. Thus, we could examine whether the metric implementation corresponds to its definition. We eliminated the heuristic where authors did not give a clear definition of the metrics they relied upon. However, metrics definitions have ambiguities that affect their implementations. For example, it is unclear whether the properties of an inner class should affect the metrics calculated for the outer class.

To calculate the required metrics, we used publicly available open-source metric extraction tools used by other researchers in the field. However, the MLCQ dataset encompasses many projects (792), and some metric values may have been miscalculated due to parsing errors. Indeed, while performing error 
analysis, our domain expert identified a few instances for which the number of unique words was miscalculated. As we used the tools tested and accepted by the community, we hope that the number of miscalculated metric values is insignificant.

\section{Conclusion}

In this paper, we performed ML-based detection of God Class and Long Method code smells. Our study is the first to consider using pre-trained code2vec, code2seq, and CuBERT embeddings as source code representation for this task. We compared this approach to a traditional ML-based code smell detection approach in which the source code is represented as a vector of hand-crafted code metrics. To test the effectiveness of the ML-based approach, we also included multiple metric-based heuristics as baselines. We evaluated our approach on the MLCQ dataset, the largest, manually labeled, and fully reproducible dataset for code smell detection. We also performed an in-depth error analysis to understand the advantages and limitations of the considered approaches.

Our results indicate that pre-trained code2vec and code2seq embedding do not generalize well to the task of code smell detection. On the other hand, the ML model trained on the pre-trained CUBERT embeddings outperformed all other approaches. We also found that the ML model trained using the hand-crafted code metrics outperformed heuristic metric-based approaches.

Our error analysis argued the advantages of using automatically inferred CUBERT features to represent source code over the metric-based representation for code smell detection. We found the metric extraction process time-consuming, brittle, and unable to capture the semantics needed to detect code smells as well as CUBERT features.

Our experiments also indicate that the field of ML-based code smell detection would benefit from a systematic approach to labeling code smells. Current datasets suffer from limitations that hinder the development of ML-based approaches. These limitations include issues with reproducibility, semiautomatic labeling of code smells, unrealistic code smell distribution, and the considerable disagreement between the annotators or failure to cross-check all labeled instances. Thus, we direct our future efforts towards developing a systematic approach for code smell labeling, inspired by the guidelines in Natural Language Processing.

\section{Acknowledgements}

This research was supported by the Science Fund of the Republic of Serbia, Grant No 6521051, Al-Clean CaDET.

\section{References}

Abbes, M., Khomh, F., Gueheneuc, Y. G., \& Antoniol, G. (2011, March). An empirical study of the impact of two antipatterns, blob and spaghetti code, on program comprehension. In 2011 15Th european conference on software maintenance and reengineering (pp. 181-190). IEEE.

Allamanis, M., Barr, E. T., Devanbu, P., \& Sutton, C. (2018). A survey of machine learning for big code and naturalness. ACM Computing Surveys (CSUR), 51(4), 1-37.

Alon, U., Brody, S., Levy, O., \& Yahav, E. (2018). code2seq: Generating sequences from structured representations of code. arXiv preprint arXiv:1808.01400. Implementation of code2seq provided by the authors is available at https://github.com/tech-srl/code2seq Accessed: July 27, 2021 
Alon, U., Zilberstein, M., Levy, O., \& Yahav, E. (2019). code2vec: Learning distributed representations of code. Proceedings of the ACM on Programming Languages, 3(POPL), 1-29. Implementation of code2vec provided by the authors is available at https://github.com/tech-srl/code2vec Accessed: July 27, 2021

Aniche, M. (2015). Java code metrics calculator (CK). Retrieved from https://github.com/mauricioaniche/ck/ Accessed July 27, 2021

Arumugam, L. (2020). Semantic code search using Code2Vec: A bag-of-paths model (Master's thesis, University of Waterloo).

Azeem, M. I., Palomba, F., Shi, L., \& Wang, Q. (2019). Machine learning techniques for code smell detection: A systematic literature review and meta-analysis. Information and Software Technology, 108, 115-138.

Bafandeh Mayvan, B., Rasoolzadegan, A., \& Javan Jafari, A. (2020). Bad smell detection using quality metrics and refactoring opportunities. Journal of Software: Evolution and Process, 32(8), e2255.

Bakarov, A. (2018). A survey of word embeddings evaluation methods. arXiv preprint arXiv:1801.09536.

Barbez, A., Khomh, F., \& Guéhéneuc, Y. G. (2019, September). Deep Learning Anti-patterns from Code Metrics History. In 2019 IEEE International Conference on Software Maintenance and Evolution (ICSME) (pp. 114-124). IEEE.

Ben-Nun, T., Jakobovits, A. S., \& Hoefler, T. (2018). Neural code comprehension: A learnable representation of code semantics. arXiv preprint arXiv:1806.07336.

Boutaib, S., Bechikh, S., Palomba, F., Elarbi, M., Makhlouf, M., \& Said, L. B. (2021). Code smell detection and identification in imbalanced environments. Expert Systems with Applications, 166, 114076.

Briand, L. C., Wüst, J., Ikonomovski, S. V., \& Lounis, H. (1999, May). Investigating quality factors in object-oriented designs: an industrial case study. In Proceedings of the 21st international conference on Software engineering (pp. 345-354).

Cairo, A. S., Carneiro, G., de Resende, A. M. P., \& Brito, F. (2019). The influence of god class and long method in the occurrence of bugs in two open source software projects: an exploratory study. In Proceedings of the International Conference on Software Engineering and Knowledge Engineering, SEKE (pp. 199-204).

Chen, T., \& Guestrin, C. (2016, August). Xgboost: A scalable tree boosting system. In Proceedings of the 22nd acm sigkdd international conference on knowledge discovery and data mining (pp. 785794).

Child, M., Rosner, P., \& Counsell, S. (2019). A comparison and evaluation of variants in the coupling between objects metric. Journal of Systems and Software, 151, 120-132.

Coimbra, D., Reis, S., Abreu, R., Păsăreanu, C., \& Erdogmus, H. (2021). On using distributed representations of source code for the detection of $C$ security vulnerabilities. arXiv preprint arXiv:2106.01367. 
Compton, R., Frank, E., Patros, P., \& Koay, A. (2020, June). Embedding java classes with code2vec: Improvements from variable obfuscation. In Proceedings of the 17th International Conference on Mining Software Repositories (pp. 243-253).

Defreez, D., Thakur, A. V., \& Rubio-González, C. (2018). Path-based function embedding and its application to specification mining. arXiv preprint arXiv:1802.07779.

Devlin, J., Chang, M. W., Lee, K., \& Toutanova, K. (2018). Bert: Pre-training of deep bidirectional transformers for language understanding. arXiv preprint arXiv:1810.04805.

Di Nucci, D., Palomba, F., Tamburri, D. A., Serebrenik, A., \& De Lucia, A. (2018, March). Detecting code smells using machine learning techniques: are we there yet?. In 2018 ieee 25th international conference on software analysis, evolution and reengineering (saner) (pp. 612-621). IEEE.

Feng, Z., Guo, D., Tang, D., Duan, N., Feng, X., Gong, M., ... \& Zhou, M. (2020). Codebert: A pre-trained model for programming and natural languages. arXiv preprint arXiv:2002.08155.

Fontana, F. A., Mäntylä, M. V., Zanoni, M., \& Marino, A. (2016). Comparing and experimenting machine learning techniques for code smell detection. Empirical Software Engineering, 21(3), 1143-1191.

Fowler, M. (2018). Refactoring: improving the design of existing code. Addison-Wesley Professional.

Hoang, T., Kang, H. J., Lo, D., \& Lawall, J. (2020, June). Cc2vec: Distributed representations of code changes. In Proceedings of the ACM/IEEE 42nd International Conference on Software Engineering (pp. 518-529).

Hovy, E. (2010, July). Annotation. In Proceedings of the 48th Annual Meeting of the Association for Computational Linguistics: Tutorial Abstracts.

Hozano, M., Garcia, A., Fonseca, B., \& Costa, E. (2018). Are you smelling it? Investigating how similar developers detect code smells. Information and Software Technology, 93, 130-146.

Hussain, Y., Huang, Z., Zhou, Y., \& Wang, S. (2020). Deep transfer learning for source code modeling. International Journal of Software Engineering and Knowledge Engineering, 30(05), 649-668.

Ide, N., \& Pustejovsky, J. (Eds.). (2017). Handbook of linguistic annotation (Vol. 1). Berlin: Springer.

Kanade, A., Maniatis, P., Balakrishnan, G., \& Shi, K. (2020, November). Learning and evaluating contextual embedding of source code. In International Conference on Machine Learning (pp. 5110-5121). PMLR. Implementation provided by the authors is available at https://github.com/google-research/google-research/tree/master/cubert Accessed: July 27, 2021

Kang, H. J., Bissyandé, T. F., \& Lo, D. (2019, November). Assessing the generalizability of code2vec token embeddings. In 2019 34th IEEE/ACM International Conference on Automated Software Engineering (ASE) (pp. 1-12). IEEE.

Karampatsis, R. M., \& Sutton, C. (2020). Scelmo: Source code embeddings from language models. arXiv preprint arXiv:2004.13214. 
Khomh, F., Di Penta, M., Guéhéneuc, Y. G., \& Antoniol, G. (2012). An exploratory study of the impact of antipatterns on class change-and fault-proneness. Empirical Software Engineering, 17(3), 243275.

Lacerda, G., Petrillo, F., Pimenta, M., \& Guéhéneuc, Y. G. (2020). Code smells and refactoring: A tertiary systematic review of challenges and observations. Journal of Systems and Software, 167, 110610.

Lemaître, G., Nogueira, F., \& Aridas, C. K. (2017). Imbalanced-learn: A python toolbox to tackle the curse of imbalanced datasets in machine learning. The Journal of Machine Learning Research, 18(1), 559-563.

Lincke, R., Lundberg, J., \& Löwe, W. (2008, July). Comparing software metrics tools. In Proceedings of the 2008 international symposium on Software testing and analysis (pp. 131-142).

Liu, H., Jin, J., Xu, Z., Bu, Y., Zou, Y., \& Zhang, L. (2019). Deep learning based code smell detection. IEEE transactions on Software Engineering.

Lozoya, R. C., Baumann, A., Sabetta, A., \& Bezzi, M. (2021). Commit2vec: Learning distributed representations of code changes. SN Computer Science, 2(3), 1-16.

Luburić, N., Prokić, S., Grujić, K. G., Slivka, J., Kovačević, A., Sladić, G., \& Vidaković, D. (2021). Towards a systematic approach to manual annotation of code smells.

[dataset] Madeyski, L., \& Lewowski, T. (2020). MLCQ: Industry-relevant code smell data set. In Proceedings of the Evaluation and Assessment in Software Engineering (pp. 342-347). Zenodo. https://zenodo.org/record/3590102\#.YG2VzegzY2w Accessed July 28, 2021

Martin, R. C. (2009). Clean code: a handbook of agile software craftsmanship. Pearson Education.

Mashhadi, E., \& Hemmati, H. (2021). Applying CodeBERT for Automated Program Repair of Java Simple Bugs. arXiv preprint arXiv:2103.11626.

Mikolov, T., Chen, K., Corrado, G., \& Dean, J. (2013). Efficient estimation of word representations in vector space. arXiv preprint arXiv:1301.3781.

Neamtiu, I., Foster, J. S., \& Hicks, M. (2005, May). Understanding source code evolution using abstract syntax tree matching. In Proceedings of the 2005 international workshop on Mining software repositories (pp. 1-5).

Palomba, F., Bavota, G., Di Penta, M., Oliveto, R., Poshyvanyk, D., \& De Lucia, A. (2014). Mining version histories for detecting code smells. IEEE Transactions on Software Engineering, 41(5), 462-489.

Palomba, F., Di Nucci, D., Tufano, M., Bavota, G., Oliveto, R., Poshyvanyk, D., \& De Lucia, A. (2015, May). Landfill: An open dataset of code smells with public evaluation. In 2015 IEEE/ACM 12th Working Conference on Mining Software Repositories (pp. 482-485). IEEE.

Palomba, F., Bavota, G., Di Penta, M., Fasano, F., Oliveto, R., \& De Lucia, A. (2018a). On the diffuseness and the impact on maintainability of code smells: a large scale empirical investigation. Empirical Software Engineering, 23(3), 1188-1221. 
Palomba, F., Bavota, G., Di Penta, M., Fasano, F., Oliveto, R., \& De Lucia, A. (2018b). A large-scale empirical study on the lifecycle of code smell co-occurrences. Information and Software Technology, 99, 1-10.

Pan, C., Lu, M., \& Xu, B. (2021). An Empirical Study on Software Defect Prediction Using CodeBERT Model. Applied Sciences, 11(11), 4793.

de Paulo Sobrinho, E. V., De Lucia, A., \& de Almeida Maia, M. (2018). A systematic literature review on bad smells -5 W's: which, when, what, who, where. IEEE Transactions on Software Engineering.

Pecorelli, F., Di Nucci, D., De Roover, C., \& De Lucia, A. (2020). A large empirical assessment of the role of data balancing in machine-learning-based code smell detection. Journal of Systems and Software, 169, 110693.

Pedregosa, F., Varoquaux, G., Gramfort, A., Michel, V., Thirion, B., Grisel, O., ... \& Duchesnay, E. (2011). Scikit-learn: Machine learning in Python. the Journal of machine Learning research, 12, 28252830.

Peters, M. E., Neumann, M., Iyyer, M., Gardner, M., Clark, C., Lee, K., \& Zettlemoyer, L. (2018). Deep contextualized word representations. arXiv preprint arXiv:1802.05365.

Piotrowski, P., \& Madeyski, L. (2020). Software defect prediction using bad code smells: A systematic literature review. Data-Centric Business and Applications, 77-99.

Pour, M. V., Li, Z., Ma, L., \& Hemmati, H. (2021, April). A Search-Based Testing Framework for Deep Neural Networks of Source Code Embedding. In 2021 14th IEEE Conference on Software Testing, Verification and Validation (ICST) (pp. 36-46). IEEE.

Rabin, M. R. I., Mukherjee, A., Gnawali, O., \& Alipour, M. A. (2020, November). Towards demystifying dimensions of source code embeddings. In Proceedings of the 1st ACM SIGSOFT International Workshop on Representation Learning for Software Engineering and Program Languages (pp. 29-38).

Rabin, M. R. I., Bui, N. D., Wang, K., Yu, Y., Jiang, L., \& Alipour, M. A. (2021). On the generalizability of Neural Program Models with respect to semantic-preserving program transformations. Information and Software Technology, 135, 106552.

Rasool, G., \& Arshad, Z. (2015). A review of code smell mining techniques. Journal of Software: Evolution and Process, 27(11), 867-895.

Sabetta, A., \& Bezzi, M. (2018, September). A practical approach to the automatic classification of security-relevant commits. In 2018 IEEE International conference on software maintenance and evolution (ICSME) (pp. 579-582). IEEE.

Sharma, T., \& Spinellis, D. (2018). A survey on software smells. Journal of Systems and Software, 138, 158-173.

Vaswani, A., Shazeer, N., Parmar, N., Uszkoreit, J., Jones, L., Gomez, A. N., ... \& Polosukhin, I. (2017). Attention is all you need. In Advances in neural information processing systems (pp. 5998-6008). 
Yamashita, A., \& Moonen, L. (2013, May). Exploring the impact of inter-smell relations on software maintainability: An empirical study. In 2013 35th International Conference on Software Engineering (ICSE) (pp. 682-691). IEEE.

Wang, S., Huang, L., Ge, J., Zhang, T., Feng, H., Li, M., ... \& Ng, V. (2020). Synergy between Machine/Deep Learning and Software Engineering: How Far Are We?. arXiv preprint arXiv:2008.05515.

Wang, Y., Gao, F., \& Wang, L. (2021). Demystifying code summarization models. arXiv preprint arXiv:2102.04625.

Wu, J. (2021). Literature review on vulnerability detection using NLP technology. arXiv preprint arXiv:2104.11230. 